\title{
Analysis of prescription frequency of herbs in traditional Korean medicine hospital using electronic medical records
}

\author{
Byung-Wook Lee ${ }^{1,2}$, Hyun-Woo Cho ${ }^{3}$, Eui-Hyoung Hwang ${ }^{4,5}$, In $\mathrm{Heo}^{4,5}$, \\ Byung-Cheul Shin ${ }^{4,5}$, Man-Suk Hwang ${ }^{4,5^{*}}$ \\ ${ }^{1}$ Department of Medical Classics and History, College of Korean Medicine, Dongguk University \\ ${ }^{2}$ Dongje Medical Co. Ltd., Republic of Korea \\ ${ }^{3}$ Department of Korean Medical Science, School of Korean Medicine, Pusan National University \\ ${ }^{4} 3^{\text {rd }}$ Division of Clinical Medicine, School of Korean Medicine, Pusan National University \\ ${ }^{5}$ Department of Rehabilitation medicine of Korean Medicine, Pusan National University Korean Medicine Hospital
}

Objectives: To analyze the prescription frequency of various herbs as either individual or major herbs (in terms of dosage) and their usage patterns in the treatment of different diseases for standardization of traditional Korean medicine.

Methods: We analyzed the prescription database of patients at the Pusan National University Korean Medicine Hospital from the date of establishment of the hospital to February 2013. The complete prescription data were extracted from the electronic medical records of patients, and the prescription frequencies of individual herbs, particularly, of major herbs, were analyzed in terms of gender, age, and international classification of diseases (ICD) code.

Results: The prescription frequency of individual herbs based on age and gender showed a similar pattern. Herbal mixtures were also distributed in a similar manner. The use of some herbs differed according to age and gender (Table 1.). The herbs that were used at high frequencies for a given ICD code had similar usage patterns in different categories. However, some major herbs in the "Jun (King)" category were used uniquely for a given ICD code (Table 2.). There was significant difference between male and female on ICD code E and N, but the other ICD codes had small differences. The ratio of herbal medicine by gender showed different usage patterns in each gender.

Conclusions: The findings of our study provide fundamental data that reflect the real clinical conditions in South Korea, and therefore, can contribute to the standardization of TKM.

$\overline{\text { Key Words }}$ : Herbal medicine, Traditional Korean medicine, Electronic medical record, prescription frequency of herbs, Clinical Data Warehouse

\section{Introduction}

Generally, an herbal medicinal formula comprises 1-40 herbs formulated based on the "Jun (King), Chen (Vassal), Zuo (Assistant), and Shi (Delivery servant)" combination theory ${ }^{1,2)}$. Patients are not commonly prescribed the same formula for the same disease. However, a basic formula is established by traditional Korean medicine (TKM) doctors and then, the herbs are added depending on the disease pattern (Korean medical diagnosis) of individuals. Hence, the majority of

\footnotetext{
- Received : 16 October 2019

- Revised : 14 November 2019

- Accepted : 21 November 2019

- Correspondence to : Man-Suk Hwang

3rd Division of Clinical Medicine, School of Korean Medicine, Pusan National University, Yangsan 50612, Republic of Korea

Tel : +82-55-360-5970, Fax : +82-51-510-8437, E-mail : hwangmansuk@gmail.com
} 
TKM doctors refer to basic formulas based on the "Dong-Ui-Bo-Gam" or "Bang-Yak-Hap-Pyeon" principles that have been in use since $1885^{3,4)}$.

The pattern identification (or syndrome differentiation) of each patient is considered before prescribing an individualized herbal medicine $^{5)}$, and TKM doctors prescribe different herbal medicines to each patient. To facilitate the manufacture of regular herbal formulas, it is important that prescriptions be standardized.

In this study, we attempted to implement the results of this study to standardize herbal medicines using electronic medical records (EMRs) in South Korea for the first time. Owing to the vastness of this database, we have reported the prescription patterns in two separate articles. Our previous article comprised a prescription analysis of raw herbal formulas ${ }^{6}$, including almost all decoction types used in South Korea. When prescribing herbal medicine to a patient, in some cases, prescription may be selected mainly on the basis of individual herbs; therefore, in the present study we focused on individual herbs. We aimed to analyze the frequency of use of individual herbs prescribed based on disease patterns identified by TKM doctors and to implement the results of this study to standardize herbal medicines.

\section{Methods}

\section{Ethics}

The study protocol was reviewed and approved by the Institutional Review Board of the Pusan National University Korean Medicine Hospital (PNUKMH, IRB approval No. KCRC-IRB-2013002, February 28, 2013). Patient confidentiality was maintained, and only clinically relevant information was used in the analysis. Individual identifiable information was excluded under the supervision of the medical record administrator.

\section{Data collection from EMR database}

The PNUKMH was established in March 2010; its EMR system allowed it to become the first national university hospital in South Korea to facilitate clinical research on TKM. The advantages of the EMR system are as follows: (1) ability to analyze prescription data in real clinical circumstances (from the date of establishment of the hospital) using the Clinical Data Warehouse (CDW) and (2) it reflects the current clinical situation of TKM practices in South Korea.

In this study, we reviewed the herbal medicine prescriptions of all patients at the PNUKMH from May 192010 (the date of establishment of the hospital) to February 28 2013. The prescription data of all inpatients and outpatients in the first three years of hospital existence (March 2010 to February 2013) were extracted from the EMR system using the CDW following predefined categories in accordance with the study protocol. A specialized medical record research coordinator extracted data by conducting a predefined query. Patient names, phone numbers, dates of birth, resident registration numbers, hospital numbers, and addresses were excluded to protect patient identity, and only clinically relevant information that was essential for the analysis was extracted for each patient namely, gender, age, International Classification of Diseases (ICD) code, prescribed herbal medicine, and individual herbs.

Although certain types of prescriptions are standardized to some extent, different types of 
clinical prescriptions, such as decoctions, granules, pills, injections, and patches, are used. Conversely, only raw herbal medicines (decoction-style) are utilized in TKM, and they are prescribed by TKM doctors based on pattern identification and syndrome differentiation. This study was performed with the aim of standardizing herbal medicines.

In TKM theory, certain herbs present in the herbal medicine formula have greater effects. In this so-called formulation theory of TKM, "Jun (King), Chen (Vassal), Zuo (Assistant), and Shi (Delivery servant)" is the universal method to prepare an herbal formula". Generally, "Jun (King)" is the most important herb in each herbal formula and is considered a major herb in terms of dosage ${ }^{1)}$. Therefore, herb with a large amount of used in prescription was regarded as a major herb, the frequency of use of individual herbs and major herb were considered separately. The total 5 most common herbs, were analyzed separately divided into 4 groups according to the theory of "Jun - Chen - Zuo - Shi" to know the differences with the role in the prescriptions and 3 groups according to the period of life (youth, adulthood and advanced age).

\section{Data analysis}

Data from the CDW related to all raw herbal prescriptions were imported into the Access program (Microsoft Korea Inc., Seoul, South Korea) and sorted as separate extracted variables predefined by the protocol. All analyses were executed by an informatics specialist (B.W.L.). Microsoft Office Excel 2013 (Microsoft Korea Inc.) was used for all statistical analyses. All categorical data are described as frequencies (\%) or ratios.
To evaluate the differences in prescriptions according to age, we divided the patients into the following three age groups: youth ( $\leq 19$ years), adult (20-60 years), and advanced age ( $\geq 60$ years).

In this study, we sought to analyze (1) the tendencies of TKM practitioners to prescribe individual herbs, and (2) whether the prescriptions differed with age, gender, and disease (ICD code). We analyzed 399,345 prescriptions over a 3-year period.

\section{Results}

The total number of prescriptions for men and women were 137,563 and 261,782 , respectively.

\section{Herb usage according to age and gender}

Regarding the prescription frequency of individual herbs based on gender, Glycyrrhizae Radix, Poria, Zingiberis Rhizoma Crudus, Citri Pericarpium, Atractylodis Rhizoma Alba, Jujubae Fructus, Paeoniae Radix, Ginseng Radix, and Rehmanniae Radix Preparata were the most commonly prescribed herbs. The prescription frequency was similar for the different age categories and genders. The aforementioned herbs were included in the 15 frequently prescribed herbs (Fig. 1). However, Cyperi Rhizoma was used more commonly in women and Astragali Radix was more commonly prescribed for men.

Zingiberis Rhizoma Crudus, Citri Pericarpium, Poria, Atractylodis Rhizoma Alba, Jujubae Fructus, Paeoniae Radix, and Glycyrrhizae Radix were commonly prescribed as major herbs. These herbs differed between genders, but their frequencies were more evenly distributed than those of the individual herbs were. Cyperi 


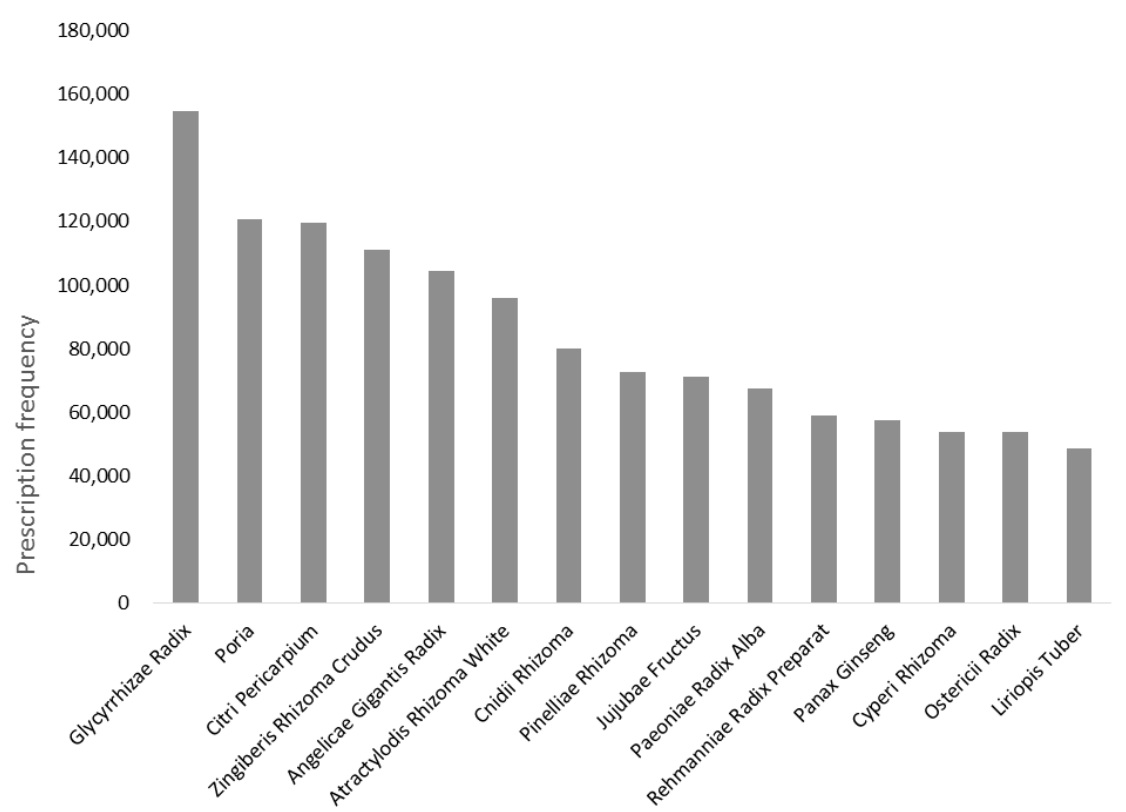

Fig. 1. Rank of herbs according to the total frequency of each herb in prescription

Rhizoma, Zizyphi Spinosae Semen, and Salviae Miltiorrhizae Radix were common in women, and Astragali Radix, Rehmanniae Radix Preparata, and Ginseng Radix, which were common in men (Table 1).

Regarding the prescription frequency of individual herbs based on age, Glycyrrhizae Radix, Citri Pericarpium, Zingiberis Rhizoma Crudus, Poria, Jujubae Fructus, Paeoniae Radix, Atractylodis Rhizoma Alba, and Ginseng Radix were the commonly prescribed individual herbs. However, there were small differences in their distribution between different age groups. Platycodi Radix and Liriopis Tuber were commonly prescribed in the "youth" group, Rehmanniae Radix Preparata and Cyperi Rhizoma were common in the "adult" group, and Rehmanniae Radix Preparata and Notopterygii Rhizoma were common in the "advanced age" group.
Regarding the prescription frequency of various herbs as major herbs according to age, Poria, Citri Pericarpium, Atractylodis Rhizoma Alba, Zingiberis Rhizoma Crudus, Paeoniae Radix, Astragali Radix, Jujubae Fructus, Zizyphi Spinosae Semen, and Cyperi Rhizoma were generally common. There were differences in their distribution between the different age groups. Puerariae Radix was common in the "youth" group, Glycyrrhizae Radix was common in the "adult" group, and Rehmanniae Radix Preparata was common in the "advanced age" group (Table $1)$.

\section{Herb usage according to ICD code \\ Regarding the prescription frequency of individual herbs according to ICD code, Glycyrrhizae Radix, Zingiberis rhizome Crudus, Poria, Citri Pericarpium, and Angelicae Gigantis}


Table 1. The five most commonly prescribed herbs used as individual or major herbs according to patient age and gender

\begin{tabular}{|c|c|c|c|c|c|}
\hline & & \multicolumn{2}{|c|}{ Prescription frequency of individual herbs } & \multicolumn{2}{|c|}{ Prescription frequency of major herbs } \\
\hline Age & Rank & & $(\%)$ & & $(\%)$ \\
\hline \multirow[t]{5}{*}{$\leq 19)$} & 1 & Glycyrrhizae Radix & 66.5 & Poria & 28.1 \\
\hline & 2 & Citri Pericarpium & 41.6 & Citri Pericarpium & 27.7 \\
\hline & 3 & Zingiberis Rhizoma Crudus & 39.5 & Atractylodis Rhizoma Alba & 25.9 \\
\hline & 4 & Poria & 37.2 & Zingiberis Rhizoma Crudus & 25.3 \\
\hline & 5 & Jujubae Fructus & 34.9 & Paeoniae Radix & 22.5 \\
\hline \multirow[t]{5}{*}{$20-59$} & 1 & Glycyrrhizae Radix & 74.5 & Zingiberis Rhizoma Crudus & 49.2 \\
\hline & 2 & Zingiberis Rhizoma Crudus & 57.0 & Atractylodis Rhizoma Alba & 29.6 \\
\hline & 3 & Poria & 53.8 & Poria & 25.1 \\
\hline & 4 & Citri Pericarpium & 51.9 & Citri Pericarpium & 24.9 \\
\hline & 5 & Atractylodis Rhizoma Alba & 47.1 & Jujubae Fructus & 24.6 \\
\hline \multirow[t]{5}{*}{$60 \geq$} & 1 & Glycyrrhizae Radix & 76.9 & Zingiberis Rhizoma Crudus & 47.8 \\
\hline & 2 & Poria & 66.9 & Poria & 35.0 \\
\hline & 3 & Zingiberis Rhizoma Crudus & 54.6 & Citri Pericarpium & 30.7 \\
\hline & 4 & Citri Pericarpium & 53.5 & Atractylodis Rhizoma Alba & 30.1 \\
\hline & 5 & Atractylodis Rhizoma Alba & 46.5 & Paeoniae Radix & 24.7 \\
\hline Gender & Rank & & $(\%)$ & & $(\%)$ \\
\hline \multirow[t]{5}{*}{ Male } & 1 & Glycyrrhizae Radix & 74.6 & Zingiberis Rhizoma Crudus & 42.6 \\
\hline & 2 & Poria & 54.4 & Atractylodis Rhizoma Alba & 32.0 \\
\hline & 3 & Citri Pericarpium & 52.6 & Poria & 28.7 \\
\hline & 4 & Zingiberis Rhizoma Crudus & 50.8 & Astragali Radix & 25.5 \\
\hline & 5 & Atractylodis Rhizoma Alba & 45.9 & Paeoniae Radix & 25.0 \\
\hline \multirow[t]{5}{*}{ Female } & 1 & Glycyrrhizae Radix & 75.6 & Zingiberis Rhizoma Crudus & 51.4 \\
\hline & 2 & Poria & 60.3 & Citri Pericarpium & 28.8 \\
\hline & 3 & Zingiberis Rhizoma Crudus & 58.6 & Poria & 28.7 \\
\hline & 4 & Citri Pericarpium & 52.1 & Atractylodis Rhizoma Alba & 28.3 \\
\hline & 5 & Atractylodis Rhizoma Alba & 46.9 & Cyperi Rhizoma & 25.8 \\
\hline
\end{tabular}

Radix were the most frequently prescribed herbs for all ICD codes. In particular, Glycyrrhizae Radix and Poria were commonly prescribed in almost all disease categories. Different herbs were prescribed for different disease categories as follows: Liriopis Tuber for endocrine, nutritional, and metabolic diseases (ICD codes: E00-E90), Bupleuri Radix for mental and behavioral disorders (F00-F99), Platycodi Radix for diseases of the respiratory system (J00-J99), Alismatis Rhizoma for diseases of the digestive system (K00-K93), Ginseng Radix for diseases of the genitourinary system (N00-N99), Cnidii Rhizoma for pregnancy, childbirth, and puerperium diseases
(O00-O99), Pinelliae Tuber for symptoms, signs, and abnormal clinical and laboratory findings not classified elsewhere (R00-R99), and Salviae Miltiorrhizae Radix for factors influencing the health status and contact with health services (Z00-Z99). For rare diseases (category Q), the prescription frequency was the same for all individual herbs (Table 2).

Regarding the frequency of herbs used as major herbs, Zingiberis Rhizoma Crudus was prescribed for almost all categories; and this tendency was as similar as the result of the total frequencies of its use as individual herb. Zingiberis Rhizoma Crudus was the most 
commonly prescribed herb, followed by Rehmanniae Radix Preparata, Cyperi Rhizoma, and Astragali Radix (Table 2). However, upon further examination, certain differences in the prescription frequency among these herbs were evident. The various herbs that were used as major herbs for different diseases are presented in Table 2.

Regarding the prescription frequency of most common herbs, there was significant difference between male and female on ICD code $\mathrm{E}$ and N, but the other ICD codes had small differences. On ICD code E, Atractylodis Rhizoma White and

Table 2. The five most commonly prescribed herbs used as individual or major herbs according to international classification of diseases code

\begin{tabular}{|c|c|c|c|c|c|}
\hline $\begin{array}{l}\text { ICD codes } \\
\text { Title }\end{array}$ & Rank & $\begin{array}{l}\text { Prescription frequency of } \\
\text { individual herbs }\end{array}$ & $(\%)$ & $\begin{array}{l}\text { Prescription frequency of major } \\
\text { herbs }\end{array}$ & $(\%)$ \\
\hline \multirow{5}{*}{$\begin{array}{l}\text { A00-B99Certain infectious and } \\
\text { parasitic diseases }\end{array}$} & 1 & Glycyrrhizae Radix & 6.56 & Zingiberis Rhizoma Crudus & 11.86 \\
\hline & 2 & Zingiberis Rhizoma Crudus & 5.01 & Citri Pericarpium & 7.15 \\
\hline & 3 & Poria & 4.91 & Poria & 6.79 \\
\hline & 4 & Citri Pericarpium & 4.16 & Pinelliae Tuber & 6.58 \\
\hline & 5 & Angelicae Gigantis Radix & 3.69 & Paeoniae Radix & 6.05 \\
\hline \multirow{5}{*}{$\begin{array}{l}\text { C00-D48 } \\
\text { Neoplasms }\end{array}$} & 1 & Glycyrrhizae Radix & 5.18 & Zingiberis Rhizoma Crudus & 12.56 \\
\hline & 2 & Atractylodis Rhizoma & 3.78 & Astragali Radix & 9.59 \\
\hline & 3 & Poria & 3.78 & Rehmanniae Radix Preparata & 7.67 \\
\hline & 4 & Zingiberis Rhizoma Crudus & 3.65 & Cyperi Rhizoma & 5.18 \\
\hline & 5 & Citri Pericarpium & 2.92 & Dioscoreae Rhizoma & 4.42 \\
\hline \multicolumn{6}{|l|}{$\begin{array}{l}\text { Diseases of the blood and } \\
\text { blood-forming organs and certain } \\
\text { disorders involving the immune } \\
\text { system }\end{array}$} \\
\hline \multirow{5}{*}{$\begin{array}{l}\text { E00-E90 } \\
\text { Endocrine, nutritional, and } \\
\text { metabolic diseases }\end{array}$} & 1 & Poria & 4.80 & Nelumbinis Semen & 10.81 \\
\hline & 2 & Glycyrrhizae Radix & 4.26 & Dioscoreae Rhizoma & 7.42 \\
\hline & 3 & Citri Pericarpium & 3.70 & Zizyphi Spinosae Semen & 6.54 \\
\hline & 4 & Atractylodis Rhizoma & 3.00 & Liriopis Tuber & 6.21 \\
\hline & 5 & Liriopis Tuber & 2.95 & Alismatis Rhizoma & 5.08 \\
\hline \multirow{5}{*}{$\begin{array}{l}\text { F00-F99 } \\
\text { Mental and behavioral disorders }\end{array}$} & 1 & Glycyrrhizae Radix & 4.13 & Rehmanniae Radix Preparata & 16.24 \\
\hline & 2 & Atractylodis Rhizoma & 3.24 & Zingiberis Rhizoma Crudus & 12.20 \\
\hline & 3 & Poria & 3.24 & Zizyphi Spinosae Semen & 10.11 \\
\hline & 4 & Angelicae Gigantis Radix & 3.10 & Pueraiae Radix & 7.79 \\
\hline & 5 & Bupleuri Radix & 3.07 & Angelicae Gigantis Radix & 7.69 \\
\hline \multirow{5}{*}{$\begin{array}{l}\text { G00-G99 } \\
\text { Diseases of the nervous system }\end{array}$} & 1 & Glycyrrhizae Radix & 4.42 & Zingiberis Rhizoma Crudus & 8.01 \\
\hline & 2 & Citri Pericarpium & 3.72 & Angelicae Gigantis Radix & 6.15 \\
\hline & 3 & Angelicae Gigantis Radix & 3.26 & Paeoniae Radix & 5.67 \\
\hline & 4 & Poria & 3.12 & Astragali Radix & 4.87 \\
\hline & 5 & Paeoniae Radix & 2.88 & Pueraiae Radix & 4.79 \\
\hline \multirow{5}{*}{$\begin{array}{l}\text { H00-H59 } \\
\text { Diseases of the eye and adnexa }\end{array}$} & 1 & Glycyrrhizae Radix & 5.39 & Astragali Radix & 11.74 \\
\hline & 2 & Poria & 3.94 & Zingiberis Rhizoma Crudus & 8.14 \\
\hline & 3 & Zingiberis Rhizoma Crudus & 3.86 & Liriopis Tuber & 6.83 \\
\hline & 4 & Citri Pericarpium & 3.82 & Lonicerae Flos & 6.38 \\
\hline & 5 & Angelicae Gigantis Radix & 3.37 & Paeoniae Radix & 4.85 \\
\hline
\end{tabular}


Table 2. The five most commonly prescribed herbs used as individual or major herbs according to international classification of diseases code

\begin{tabular}{|c|c|c|c|c|c|}
\hline $\begin{array}{l}\text { ICD codes } \\
\text { Title }\end{array}$ & Rank & $\begin{array}{l}\text { Prescription frequency of } \\
\text { individual herbs }\end{array}$ & $(\%)$ & $\begin{array}{l}\text { Prescription frequency of major } \\
\text { herbs }\end{array}$ & $(\%)$ \\
\hline \multirow{5}{*}{$\begin{array}{l}\text { H60- H95 } \\
\text { Diseases of the ear and mastoid } \\
\text { process }\end{array}$} & 1 & Glycyrrhizae Radix & 5.39 & Zingiberis Rhizoma Crudus & 11.15 \\
\hline & 2 & Poria & 3.94 & Cyperi Rhizoma & 9.27 \\
\hline & 3 & Zingiberis Rhizoma Crudus & 3.86 & Bupleuri Radix & 8.26 \\
\hline & 4 & Citri Pericarpium & 3.82 & Pueraiae Radix & 6.68 \\
\hline & 5 & Angelicae Gigantis Radix & 3.37 & Nelumbinis Semen & 5.81 \\
\hline \multirow{5}{*}{$\begin{array}{l}\text { I00-I99 } \\
\text { Diseases of the circulatory system }\end{array}$} & 1 & Glycyrrhizae Radix & 4.76 & Zingiberis Rhizoma Crudus & 16.67 \\
\hline & 2 & Citri Pericarpium & 3.98 & Astragali Radix & 5.96 \\
\hline & 3 & Poria & 3.83 & Rehmanniae Radix Preparata & 4.17 \\
\hline & 4 & Zingiberis Rhizoma Crudus & 3.59 & Angelicae Gigantis Radix & 4.15 \\
\hline & 5 & Angelicae Gigantis Radix & 3.35 & Paeoniae Radix & 3.88 \\
\hline \multirow{5}{*}{$\begin{array}{l}\text { J00-J99 } \\
\text { Diseases of the respiratory system }\end{array}$} & 1 & Glycyrrhizae Radix & 5.78 & Liriopis Tuber & 7.21 \\
\hline & 2 & Poria & 3.91 & Rehmanniae Radix Preparata & 7.11 \\
\hline & 3 & Pinelliae Tuber & 3.29 & Poria & 6.81 \\
\hline & 4 & Citri Pericarpium & 3.28 & Astragali Radix & 6.11 \\
\hline & 5 & Platycodi Radix & 3.06 & Pinelliae Tuber & 5.15 \\
\hline \multirow{5}{*}{$\begin{array}{l}\text { K00-K93 } \\
\text { Diseases of the digestive system }\end{array}$} & 1 & Poria & 4.89 & Rehmanniae Radix & 10.55 \\
\hline & 2 & Glycyrrhizae Radix & 3.99 & Rehmanniae Radix Preparata & 9.37 \\
\hline & 3 & Citri Pericarpium & 3.92 & Artemisiae capillaris herba & 6.08 \\
\hline & 4 & Zingiberis Rhizoma Crudus & 3.05 & Zingiberis Rhizoma Crudus & 6.02 \\
\hline & 5 & Alismatis Rhizoma & 3.05 & Pinelliae Tuber & 5.79 \\
\hline \multirow{5}{*}{$\begin{array}{l}\text { L00-L99 } \\
\text { Diseases of the skin and } \\
\text { subcutaneous tissue }\end{array}$} & 1 & Glycyrrhizae Radix & 6.39 & Astragali Radix & 6.42 \\
\hline & 2 & Poria & 3.95 & Poria & 5.50 \\
\hline & 3 & Angelicae Gigantis Radix & 3.88 & Angelicae Gigantis Radix & 5.37 \\
\hline & 4 & Zingiberis Rhizoma Crudus & 3.69 & Atractylodis Rhizoma Alba & 5.16 \\
\hline & 5 & Jujubae Fructus & 2.88 & Glycyrrhizae Radix & 5.09 \\
\hline \multirow{5}{*}{$\begin{array}{l}\text { M00-M99 } \\
\text { Diseases of the musculoskeletal } \\
\text { system and connective tissue }\end{array}$} & 1 & Glycyrrhizae Radix & 4.64 & Zingiberis Rhizoma Crudus & 6.69 \\
\hline & 2 & Angelicae Gigantis Radix & 3.43 & Cyperi Rhizoma & 6.62 \\
\hline & 3 & Zingiberis Rhizoma Crudus & 3.32 & Pueraiae Radix & 5.09 \\
\hline & 4 & Poria & 3.18 & Atractylodis Rhizoma Alba & 5.06 \\
\hline & 5 & Citri Pericarpium & 3.09 & Lonicerae Flos & 4.79 \\
\hline \multirow{5}{*}{$\begin{array}{l}\text { N00-N99 } \\
\text { Diseases of the genitourinary } \\
\text { system }\end{array}$} & 1 & Glycyrrhizae Radix & 5.94 & Cyperi Rhizoma & 16.87 \\
\hline & 2 & Citri Pericarpium & 5.56 & Zingiberis Rhizoma Crudus & 15.73 \\
\hline & 3 & Poria & 5.06 & Astragali Radix & 12.23 \\
\hline & 4 & Ginseng Radix & 4.68 & Rehmanniae Radix Preparata & 7.98 \\
\hline & 5 & Atractylodis Rhizoma & 4.08 & Poria & 4.56 \\
\hline \multirow{5}{*}{$\begin{array}{l}\text { O00-O99 } \\
\text { Pregnancy, childbirth, and the } \\
\text { puerperium }\end{array}$} & 1 & Glycyrrhizae Radix & 7.65 & Atractylodis Rhizoma & 9.71 \\
\hline & 2 & Cnidii Rhizoma & 6.52 & Angelicae Gigantis Radix & 7.44 \\
\hline & 3 & Angelicae Gigantis Radix & 6.30 & Cyperi Rhizoma & 6.15 \\
\hline & 4 & Citri Pericarpium & 6.19 & Zingiberis Rhizoma Crudus & 5.83 \\
\hline & 5 & Paeoniae Radix & 5.96 & Poria & 5.50 \\
\hline \multicolumn{6}{|l|}{$\begin{array}{l}\text { Certain conditions originating in } \\
\text { the perinatal period }\end{array}$} \\
\hline \multirow{5}{*}{$\begin{array}{l}\text { Q00-Q99 } \\
\text { Congenital malformations, } \\
\text { deformations, and chromosomal } \\
\text { abnormalities }\end{array}$} & 1 & Ginseng Radix & 4.27 & Lonicerae Flos & 57.14 \\
\hline & 2 & Phellodendri cortex & 4.27 & Myrrh & 42.86 \\
\hline & 3 & ScutellariaeRadix & 4.27 & & \\
\hline & 4 & Lonicerae Flos & 4.27 & & \\
\hline & 5 & Coptidis Rhizoma & 4.27 & & \\
\hline
\end{tabular}


Table 2. The five most commonly prescribed herbs used as individual or major herbs according to international classification of diseases code

\begin{tabular}{|c|c|c|c|c|c|}
\hline $\begin{array}{l}\text { ICD codes } \\
\text { Title }\end{array}$ & Rank & $\begin{array}{l}\text { Prescription frequency of } \\
\text { individual herbs }\end{array}$ & $(\%)$ & $\begin{array}{l}\text { Prescription frequency of major } \\
\text { herbs }\end{array}$ & $(\%)$ \\
\hline \multirow{5}{*}{$\begin{array}{l}\text { R00-R99 } \\
\text { Symptoms, signs, and abnormal } \\
\text { clinical and laboratory findings; not } \\
\text { classified elsewhere }\end{array}$} & 1 & Glycyrrhizae Radix & 4.51 & RehmanniaeRadix Preparata & 5.99 \\
\hline & 2 & Poria & 3.72 & Zingiberis Rhizoma Crudus & 5.91 \\
\hline & 3 & Citri Pericarpium & 3.51 & Zizyphi Spinosae Semen & 4.82 \\
\hline & 4 & Zingiberis Rhizoma Crudus & 2.72 & Pinelliae Tuber & 4.41 \\
\hline & 5 & Pinelliae Tuber & 2.53 & Astragali Radix & 4.33 \\
\hline \multirow{5}{*}{$\begin{array}{l}\text { S00-T98 } \\
\text { Injury, poisoning, and certain other } \\
\text { consequences of external causes }\end{array}$} & 1 & Glycyrrhizae Radix & 4.85 & Cyperi Rhizoma & 9.12 \\
\hline & 2 & Citri Pericarpium & 3.34 & Zizyphi Spinosae Semen & 7.17 \\
\hline & 3 & Atractylodis Rhizoma & 3.11 & Zingiberis Rhizoma Crudus & 7.16 \\
\hline & 4 & Zingiberis Rhizoma Crudus & 3.08 & Rehmanniae Radix Preparata & 5.61 \\
\hline & 5 & Angelicae Gigantis Radix & 3.01 & AstragaliRadix & 3.58 \\
\hline \multirow{5}{*}{$\begin{array}{l}\text { U00-U99 } \\
\text { Codes for special purposes }\end{array}$} & 1 & Poria & 4.56 & Salviae Miltiorrhizae Radix & 9.18 \\
\hline & 2 & Glycyrrhizae Radix & 4.10 & Zingiberis Rhizoma Crudus & 8.94 \\
\hline & 3 & Citri Pericarpium & 3.79 & Rehmanniae Radix Preparata & 7.21 \\
\hline & 4 & Zingiberis Rhizoma Crudus & 3.64 & Rehmanniae Radix & 6.65 \\
\hline & 5 & Atractylodis Rhizoma & 3.26 & Cyperi Rhizoma & 5.41 \\
\hline \multicolumn{6}{|l|}{$\begin{array}{l}\text { External causes of morbidity and } \\
\text { mortality }\end{array}$} \\
\hline \multirow{5}{*}{$\begin{array}{l}\text { Z00-Z99 } \\
\text { Factors influencing health status } \\
\text { and contact with health services }\end{array}$} & 1 & Angelicae Gigantis Radix & 5.99 & Salviae Miltiorrhizae Radix & 60.67 \\
\hline & 2 & Glycyrrhizae Radix & 5.95 & Lonicerae Flos & 9.55 \\
\hline & 3 & Atractylodis Rhizoma & 5.39 & Forsythia Suspensa & 9.55 \\
\hline & 4 & Citri Pericarpium & 5.35 & Jujubae Fructus & 6.74 \\
\hline & 5 & Salviae Miltiorrhizae Radix & 5.35 & Castaneae Semen & 3.93 \\
\hline
\end{tabular}

Rehmanniae Radix Preparata were mostly used as "Jun" in both genders, but the others were great different. Glycyrrhizae Radix, Paeoniae Radix Alba, Panax Ginseng Radix and Astragali Radix were more used in male than female but Jujubae Fructus, Poria, Zingiberis Rhizoma Crudus, Citri Pericarpium and Cyperi Rhizoma were in complete opposition to the former. The most herbs were used as "Jun", but Jujubae Fructus in male, Paeoniae Radix Alba in female, Zingiberis Rhizoma Crudus in male and Citri Pericarpium in male were more used as "Chen" and Glycyrrhizae Radix was more used as "Zuo". On ICD code N, Atractylodis Rhizoma Alba, Zingiberis Rhizoma Crudus, Rehmanniae Radix Preparata and Cyperi Rhizoma were highly used as "Jun" in both genders, but the others showed differences. Glycyrrhizae Radix, Paeoniae Radix Alba, Poria, Panax Ginseng Radix and Citri Pericarpium were more used in male than female but Jujubae Fructus and Astragali Radix were the other way round. Also the most herbs used as "Jun" but Paeoniae Radix Alba, Poria and Citri Pericarpium in female were more used as "Chen" and Citri Pericarpium and Astragali Radix in male were more used as "Zuo" (Fig. 2A).

Regarding the total prescription number, ICD code $\mathrm{M}$ was the most used part and ICD code $\mathrm{S}-\mathrm{T}$ were similar groups as musculoskeletal diseases. There was no significant difference between male and female on ICD code $\mathrm{M}$, but ICD code S-T had certain differences. On ICD 


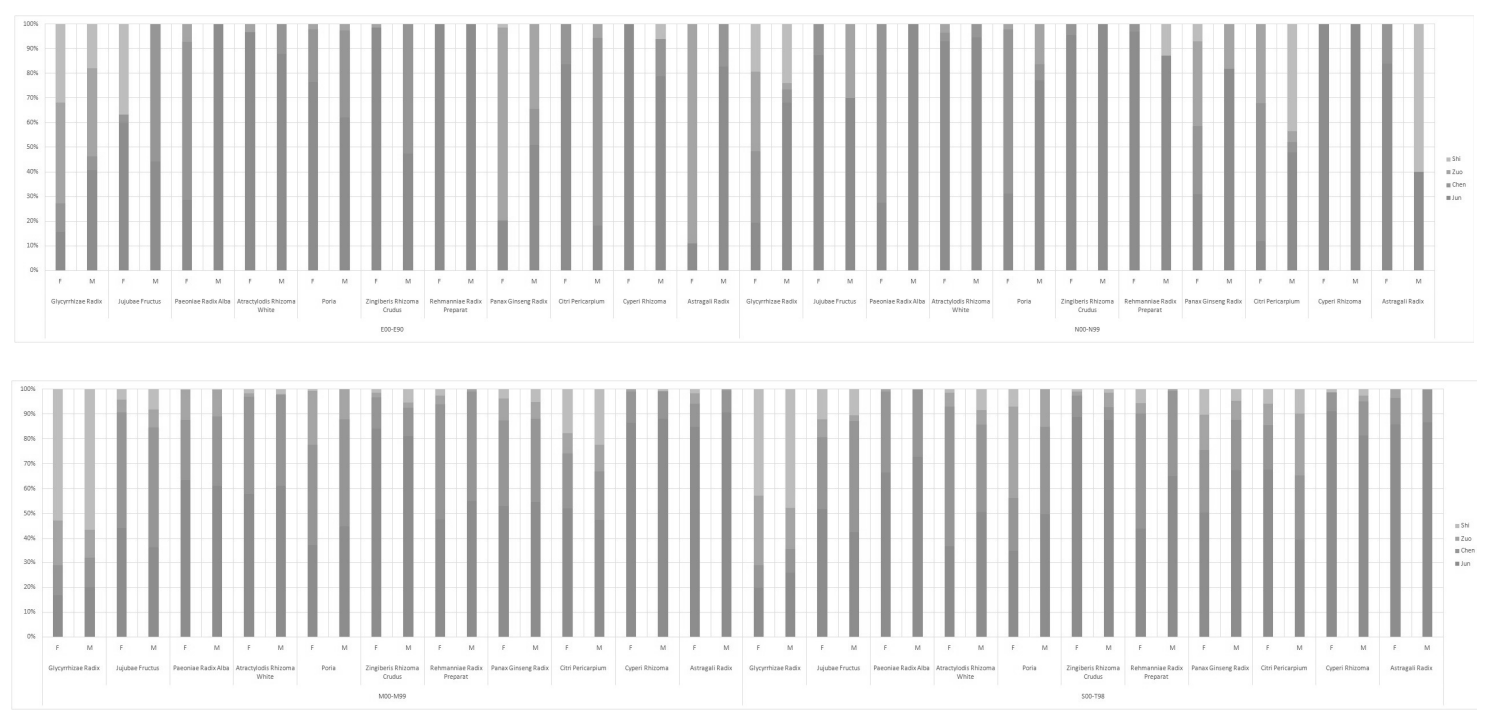

Fig. 2. Difference between male and female on ICD code; (A) ICD code $E$ and $N$, (B) ICD code $M, S$ and $T$

code S-T, Zingiberis Rhizoma Crudus and Astragali Radix were similarly used in both gender but the others were different. Atractylodis Rhizoma Alba, Poria, Rehmanniae Radix Preparata and Panax Ginseng Radix were highly more used as "Jun" in male but Jujubae Fructus, Citri Pericarpium and Cyperi Rhizoma were more used as "Jun" in female. On ICD code M, 9 herbs were mostly used as "Jun", but Jujubae Fructus was mostly used as "Chen" and Glycyrrhizae Radix was highly used as "Shi". In ICD code S-T, on the other herbs, Atractylodis Rhizoma Alba and Rehmanniae Radix Preparata in female more used as "Chen" and Glycyrrhizae Radix in both genders was more used as "Zuo" (Fig. 2B).

\section{Discussion}

Similar herbs were frequently prescribed for both genders. Glycyrrhizae Radix, Poria, and
Citri Pericarpium were used in "Erchen-tang" preparation, while Ginseng Radix, Atractylodis Rhizoma Alba, Poria, and Glycyrrhizae Radix were used in "Si Jun Zi Tang." "Erchen-Tang" is the representative decoction used to treat "phlegm pattern," which is the major cause of disease in $\mathrm{TKM}^{7)}$. Therefore, the "phlegm pattern" and "the deficiency of qi" are considered the major reasons for diseases in both genders.

"Blood stasis" is defined as a morbid state of blood stagnancy in certain areas of the body. Salviae Miltiorrhizae Radix is often used to treat this condition. It is also used often in obstetrics and gynecology. It was frequently prescribed to all women in the present study. Astragali Radix, a popular "Tonify qi" herb in $\mathrm{TKM}^{8)}$, was frequently prescribed to men in the present study. Thus, TKM practitioners seem to consider the reasons for stress as "stagnated blood" in women and "the deficiency of qi" in men (Table 1). This tendency may be a result of the traditional 
Chinese medicine or TKM formula-combination theory. These herbs (Salviae Miltiorrhizae Radix, Astragali Radix) are generally used to detoxify, activate, or transport the components of a given Korean medicine ${ }^{2,9)}$. The aforementioned functions exist under the "Chen (Vassal)," "Zuo (Assistant)," and "Shi (Delivery servant)" categories. Although the dosages of herbs exhibiting this role are generally low, they are frequently prescribed in herbal formulas. In summary, they play a minor role and are present in nearly all prescriptions.

There were partial differences in terms of prescription frequency in the different age categories. Rehmanniae Radix Preparata, Cyperi Rhizoma, Glycyrrhizae Radix, and Notopterygii Rhizoma were used in the "adult" and "advanced age" groups, whereas Platycodi Radix, Liriopis Tuber, and Puerariae Radix were used in the "young" group. Respiratory and digestive diseases account for a large portion of primary care at a young age. "Erchen-Tang" which treats digestive diseases $^{7)}$, whereas Platycodi Radix, Liriopis Tuber, and Puerariae Radix are often used for the treatment of respiratory diseases ${ }^{10}$. Therefore, decoctions used in the young are different from those used in other age groups.

Liriopis Tuber, Pinelliae Tuber, and Salviae rbs. It is likely that these herbs are used often and have considerable effects. The uses of these herbs were examined more specifically, and it was found that Liriopis Tuber, which is often used to treat diabetes mellitus, was included in the preparations for ICD codes E00-E90 ${ }^{11)}$. Pinelliae Tuber, commonly used to treat phlegm, was included in the preparations for ICD codes R00-R99 ${ }^{12)}$. Salviae Miltiorrhizae Radix was included in preparations for ICD codes Z00-Z99, because it is used to improve cardiovascular function $^{13)}$.

Regarding the prescription frequency of individual herbs by ICD code, Bupleuri Radix, which is often used to provide psychological stability, was included in preparations for ICD codes F00-F99 ${ }^{14)}$. Platycodi Radix, which is generally used to treat respiratory diseases, was included in decoctions for ICD codes J00-99 ${ }^{10}$. Alismatis Rhizoma was included in herbal blends for ICD codes K00-93 because it is frequently used to treat diarrhea and has a diuretic effect ${ }^{10}$. Ginseng Radix, which is used to cure impotence (it acts on the blood vessels), was included in preparations for ICD codes N00-99 ${ }^{15)}$. Cnidii rhizome, which is often used to manage the function of the uterus as a component of "Gung-gui-jo-hyeol-eum," was included in preparations for ICD code O00-99 ${ }^{16}$. There were significant variations in "Jun-Chen-Zuo-Shi." The herbs in the "Zuo (Assistant)" or "Shi (Delivery servant)" categories were commonly used in prescriptions irrespective of age, gender, or disease, whereas herbs in the "Jun (King)" or "Chen (Vassal)" categories were used according to individual ICD codes. These findings indicate that prescriptions address customized symptoms, diseases, and conditions in TKM.

This study may be important in some respects. Firstly, this is the first study using current clinical data to facilitate the standardization of herbal formulas in TKM. Secondly, this study included the most standardized clinical information gathered to date, reflecting the reliability and clinical relevance.

However, there are certain limitations to our study. First, we performed the study only in one hospital; therefore, our results may not reflect all of the features of TKM in terms of regional 
differences in medical staff. To confirm some of our observations and to standardize herbal medicines accurately, studies using EMR data from several hospitals employing different styles of herbal medicines should be conducted.

In conclusion, we identified certain prescription tendencies of individual herbs according to age, gender, and disease condition using the prescription frequency of individual and major herbs. We inferred that TKM does not simply focus on individual diseases, but on case-by-case treatment according to gender, age, and diseases. Therefore, our data would be helpful to standardize prescription tendencies of TKM doctors in real practice. We plan to conduct a study comparing TKM with traditional Chinese medicine in the near future.

\section{Conflict of interest}

The authors report no conflict of interest.

\section{Acknowledgements}

This work was supported by clinical research grant from Pusan National University Hospital in 2019

\section{Contribution}

All data were extracted and analyzed by an informatics specialist (B.W.L).

B.W.L. and H.W.C. wrote the manuscript.

B.C.S. and E.H.H. compiled and organized the data, conducted the analyses, and drafted the manuscript. M.S.H. contributed to the study conception and design. E.H.H., H.Y.L., and I.H. contributed to the analysis and interpretation of the data.

English proofreading and formatting services were obtained from the Editage company.

\section{References}

1. Sheng S, Wang J, Wang L, Liu H, Li P, Liu $\mathrm{M}$, et al., Network pharmacology analyses of the antithrombotic pharmacological mechanism of Fufang Xueshuantong Capsule with experimental support using disseminated intravascular coagulation rats. Journal of ethnopharmacology, 2014;154:735-44.

2. Choi S, Chang I, A milestone in codifying the wisdom of traditional oriental medicine: TCM, Kampo, TKM, TVM-WHO international standard terminologies on traditional medicine in the western pacific region. Evid Based Complement Alternat Med, 2010;7(3):303-5.

3. Yoon CY, Kim HT, A study on Bang-Yak -Hap-Pyun. J Orient Med Class, 1991;5:151-349.

4. Kang JH, Yang DH, Park YB, Kimp S, A Text Mining Approach to Find Patterns Associated with Diseases and Herbal Materials in Oriental Medicine. IJET, 2012;2(3):224-6.

5. Kim HJ, Bae HS, Park SU, Moon SK, Park JM, Jung WS, Clinical Approach to the Standardization of Oriental Medical Diagnostic Pattern Identification in Stroke Patients. Evid Based Complement Alternat Med, 2011;2011:7.

6. Lee B-W, Lee H-Y, Heo K-H, Cho H-W, Hwang M-S, Heo I, et al., Analysis of herbal medicine prescriptions for patients in an academic Korean medical hospital: A cross sectional study of electronic medical records (2010-2013). Chinese journal of 
integrative medicine, 2017.

7. Jiang WY, Therapeutic wisdom in traditional Chinese medicine: a perspective from modern science. Trends Pharmacol Sci, 2005;26(11): 558-63.

8. Yeh TS, Chuang HL, Huang WC, Chen YM, Huang CC, Hsu MC, Astragalus membranaceus improves exercise performance and ameliorates exercise-induced fatigue in trained mice. Molecules., 2014;19(3):2793-807. doi:10.3390/ molecules 19032793 .

9. Wang L, Zhou GB, Liu P, Song JH, Liang Y, Yan XJ, et al., Dissection of mechanisms of Chinese medicinal formula Realgar-Indigo naturalis as an effective treatment for promyelocytic leukemia. Proc Natl Acad Sci U.S.A., 2008;105(12):4826-31.

10. Liu C, Tseng A, Yang S, Chinese herbal medicine: Modern applications of traditional formulas. CRC Press. 2004.

11. Rhee I, Effect of Liriopis Tuber extract on the decrease of blood glucose. Hyosung Bull. Pharm. Sci, 1997;2:49-56.

12. Wenyue J, Yu Y, Yanyan L, Effects of some drugs for resolving phlegm on blood rheological property in rats. $\mathrm{J}$ Tradit Chin
Med, 2002;3:038.

13. Cheng TO, The international textbook of cardiology. Pergamon Press. 1986.

14. Chen JX, Ji B, Lu ZL, Hu LS, Effects of Chai $\mathrm{Hu}$ (Radix Burpleuri) containing formulation on plasma $\beta$-endorphin, epinephrine and dopamine in patients. Am J Chin Med, 2005;33(05):737-45.

15. Hafez DA, Effect of extracts of ginger goots and cinnamon bark on fertility of male diabetic rats. J Am Sci, 2010;6:940-7.

16. Choi BT, Seo IB, Lee DN, Kim HJ, Inhibiting Effecposets of Gungguijohyeoleum on the Development of Experimentally -induced Endometriosis in the Rats. J Orient Obstet Gynecol, 2008;21(3):34-59.

\section{ORCID}

Byung-Wook Lee https://orcid.org/0000-0002-8413-4211 Hyun-Woo Cho https://orcid.org/0000-0002-1458-388X Eui-Hyoung Hwang https://orcid.org/0000-0003-0460-3194 In Heo https://orcid.org/0000-0003-2893-8917 Byung-Cheul Shin https://orcid.org/0000-0002-0059-2689 Man-Suk Hwang https://orcid.org/0000-0003-2154-7358 